\title{
Mechanisms of immune evasion by Trypanosoma brucei.
}

\author{
María Elizabeth Márquez-Contreras*
}

Departement of Parasite Enzymology, Faculty Science, University of the Andes, Venezuela

\begin{abstract}
Trypanosoma brucei is the parasite responsible of causing both human (sleeping sickness) and animal (nagana) African trypanosomiasis, diseases of medical and veterinary importance. The infection in cattle has a major impact on African economy, which limits the production of milk and meat. The life cycle of these parasites extracellular alternate between the salivary glands of the insect transmitter (tsetse fly) and the blood of the vertebrate (mammal), presenting different morphological stages: procyclic, epimastigote, metacyclic forms in the invertebrate, long-slender trypomastigotes and shortstumpy forms in the vertebrate. The ATs are extracellular microorganisms that multiply in the blood and the lymphatic system and are constantly exposed to the attack of the the immune system of the mammalian host. The metacyclic parasites express a dense cover formed by the Variant Surface Glycoprotein (VSG), after entering to the vertebrate host they transform into a morphology that stays in the blood (bloodstream). This is possible thanks to a fundamental mechanism developed by ATs, the switch of VSG through which the parasites change his coat regularly and liberate the VSG expressed at this time, which allows the evasion of the attack of the immune system of the vertebrate host (specifically humoral response). In addition to all these events, this pathogens have generated an activation of M1 dependent of IFN- $\gamma$; express proteins of resistance to factors of human serum, for example, SRA (Serum Resistance Antigen) and TgsGP (T. b. gambiense-specific glycoprotein); Glycosylinositolphosphate-Variable Surface Glycoprotein (GIP-VSG) produce the activation of NF-кB and MAPK pathways and the production of pro-inflammatory molecules (TNF- $\alpha$, IL-6, IL-12p40, and GM-CSF) that with IFN- $\gamma$ activated macrophages type M1, promotes a series of pathologies as tissue damage and encephalitis that provoke destruction in the Central Nervous System (CNS); these are just some of the factors that support the development of the infection in mammals. The African trypanosomes (AT) have learned to survive the attack of the defense system of vertebrates, through many years of carrying out a host-parasite interaction extremely complex and dynamic. Throughout the life cycle, these parasites re-programed your metabolism in order to benefit from the nutrients available in the environment. This abilities has allowed these escape magicians advance effectively and successfully in the development of immune system evasion mechanisms, allowing manipulating cellular and humoral responses, which implies a spectacular equilibrium in the vector/parasite/host relationship that allows their survival in the mammal and leads to a happy term their cycle of transmission.
\end{abstract}

Keywords: Immune evasion,Trypanosomiasis, Innate immune response, Antigenic variation, Humoral response.

\section{Introduction}

T. brucei is a hemoflagellate of the family Trypanosomatidae (orden Kinetoplastida), with a digenetic life cycle, that is, that develops in a vertebrate host and invertebrate host. The mammal becomes infected when the tsetse fly injects into its skin the metacyclic parasites along with the saliva, which multiply at the entry site and sometimes produce a lesion known as chancre. Subsequently, some of them are transformed to the long and slender blood-stream trypomastigotes, takes place the process of division and they become in shorter, stumpy blood-stream form of parasite. Some of these last forms are ingests by the tsetse fly during a blood meal. It is important to emphasize that these forms have a preadaptation to survive in the environmental conditions present in the insect vector [1]. They then become procyclical forms in the insect's midgut and migrate to the salivary glands where they become epimastigotes. Later, the conversion to infectious metacyclic forms occurs, which is preadapted to grow and multiply within the blood of mammals. This completes the complex life cycle of these extraordinary organisms (Figure 1) [2]. Human African trypanosomiasis (HAT) it is known as "sleeping sickness", because one of the characteristic signs of this disease is the alteration of the sleep cycle which manifests in the advanced stage of this parasitary infection [3]. HAT attacks the central nervous system and infection can be by Trypanosoma brucei gambiense in some countries of Africa (Western and Central) and Trypanosoma brucei rhodesiense in other countries of Africa (Eastern and Southern). These parasites species present identical morphological features, but their epidemio-logical characteristics are very dissimilar. Nagana and Surra is how are designated the disease of trypanosome in cattle, that produces large epidemic in Africa with high impact economical, due to the death of infected animals [4]. The co-evolution of many years between the parasite/host has allowed evading the primary responses (innates) and escaping to the first barriers 
shown by the organism, in this way the parasite implants in the host and starts the infection.

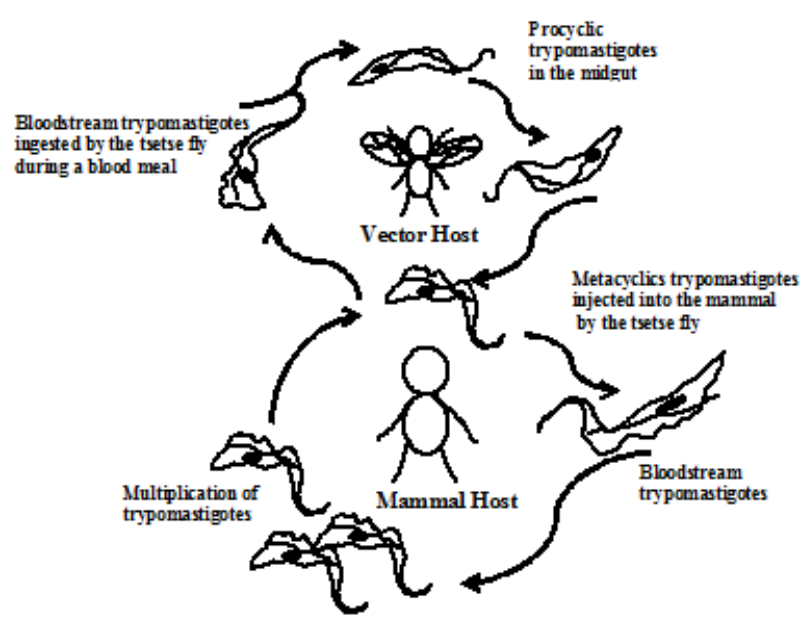

Figure 1. Life cycle of T. brucei.

Moreover, it also escapes to the immune system action throughout all its permanence in the vertebrate. The most interesting and fabulous biological mechanism of immune system disorder is the capacity of the trypanosome to change every so often the antigenic cover (VSG), which involves programmed rearrangements in the pathogen's DNA. This phenomenon originates polyclonal activation of $\mathrm{B}$ and $\mathrm{T}$ lymphocytes, as one of the host's defenses tool, related to immunosuppression, favoring the perpetuation of these parasites [5,6]. VSG can work in two ways: avoiding access of components of the immune system (innate/acquired) to invariant proteins on its surface and keeping safe the parasites in the blood of elimination by the complement system [7]. This review describes some of the mechanisms applied by these parasites in order to effect the evasion of immune system.

\section{Escape of host's defenses}

The mammals can respond immunologically through two pathways: the innate system (low specific) and the adaptive response (antigen specific).

\section{Evasion of innate response}

Theinnate immune system is the first barrier of mammals to defend themselves against trypanosomes. T. b. gambiense and T. $b$. rhodesiense are subject to the lytic action of two serum human complexes, denominated trypanosome lytic factor (TLF) (TLF 1 and TLF 2) [8], containing apolipoprotein L1 (APOL1) [9] which is endocytosed via the haptoglobinhemoglobin parasite surface receptor. Both parasites evade the destruction by APOL1 producing certain proteins that give them resistance [10]. The T. $b$. gambiense generated a TgsGP, a glycoprotein that counteracts APOL1 activity, which allows escaping from the death activated by TLF particles [11]. This protein is indispensable in order to protect against the components of human serum, because the elimination of TgsGP favors susceptibility of the parasites to human serum and recombinant APOL1, when TgsGP reenters the weakened parasite resistance reappears [12]. T. $b$. rhodesiense produces SRA, a modified form of VSG, which interacts in the lysosome with APOL1, induces resistance against normal human serum (NHS). SRA refers to an expression site-associated gene (ESAG), with a determinate VSG ES, denominated R-ES, picked in the human serum through the process of antigenic variation $[13,14]$. It has been observed that the production of SRA by $T$. $b$. rhodesiense is not permanent, since after making peals of the parasites in mice it is lost the resistance to NHS, due to lack of the trypanolytic factor of the human serum [15].

\section{Complement}

The complement activation can start through two pathways: the alternative pathway (independent of antibody) and the classical pathway (dependent of antibody). The turn on of the alternative pathway is supremely important in the destruction of the parasite during the beginning of the disease. T. $b$. gambiense trypomastigotes forms prevent the switch on of the alternative pathway of the complement, using VSGs to block the areas on the surface of the microorganism that started this immune cascade [16]. This process occurs without appearance of the final complex (C5-C9) which can produce membrane rupture (lytic activity is inhibited), this route stops at the moment that the $\mathrm{C} 3$ convertase is established in the membrane of the microorganism [17]. Besides, the infections by T. b. gambiense or $T$. $b$. rhodesiense originates a decreased in complement activity [18]. For the study of the alternative pathway, the procyclic forms of $T$. $b$. congolense and $T$. $b$. brucei were placed in the presence of fresh serum, in this experiment it is noted lysis of the trypanosomes, because in this stage of the cycle of life they don't present the protective cover of VSG [5].

The classical complement pathway switches on when the parasites (coated with VSG) are eliminated shoots out by antibody mediated lysis and/or opsonization. This process induces the formation of anaphylatoxins or proteins of complement, which are originated as products of the activation of the complement pathway; it originates vasodilatation, histamine release from mast cells, smooth muscle contraction, enhanced vascular permeability, inflammation, chemotaxis and liberation of cytotoxic radicals. The activation of the system complement is essential for the lysis of trypanosomes dependent of antibody, that occur by liberation of a numerous quantity of soluble VSG (sVSG), this phenomenon favors the decrease of elements of the complement that leads to a hypocomplementemia [19]. The immune complexes that induce complement activation are products of noncovalents interactions between antibodies specifically directed against trypanosomes (eg anti-VSG antibody) and trypanosomes antigens or autoantigen, they trigger the appearance of aggressive effects including tissue destruction [20, 21]. The bloodstream form of $T$. brucei is rich in VSG, a glycoprotein that forms a cover of $5 \times 10^{6}$ homodimers for cell that presents helix $\alpha \mathrm{A}$ and B antiparallels, attached to the exracellular face of the plasma membrane by means of glycosylphosphatidylinositol (GPI) anchor which contained mannose, glucosamine, ethanolamine and a short galactose chain [22]. These structures are dispuested with the N-terminal 
hydrophilic extreme exposed to the extracellular space and the C-terminal towards the plasmatic membrane. These composition origin a structure very ordinated and packaged on the surface of the parasite denominated VSG.

The VSG coat is a macromolecular diffusion barrier that protects the parasite from the innate immune system and also enables the parasite to undergo antigenic variation. Thus, although an individual parasite only expresses one VSG gene at a time, the parasite population can stay ahead of the host's specific immune response to expressed VSGs when a few parasites switch expression to one of several hundred genes encoding immunologically distinct VSGs. This structure is very antigenic and triggers a robust response that avoids an efficient opsonization of the bloodstream parasites, which prevents it from being destroyed by the mechanism of complemented-mediated lysis [23]. VSG present GPI anchors that can decay by parasite's endogenous phospholipase $\mathrm{C}$ (PLC), which is primarily located in the flagellar pocket and as they think, can participate in the pathway of internalization and recycling of VSG of membrane [24]. PLC hydrolyzes the GPI anchor of the membrane-bound form VSG (mfVSG), trigger the liberation of VSGs, which originates the appearance of a pro-inflammatory response, for this reason it is involved in the innate response [25]. PLC transform the membrane-form VSG (mfVSG) (hydrophobic) in sVSG (water-soluble), this event provoke the releasing of dimyristoyl glycerol (DMG) coming from the GPI-anchor and the glycosylinositolphosphate GIPVSG [26]. Evidence shows the GIP-galactose is very important in the production TNF- mediated sVSG [27]. The GIP-VSG interact with a Type A scavenger receptor produced on myeloid cells, that causes the activation of NF- $\kappa$ B and MAPK pathways and the production of pro-inflammatory molecules, such as TNF- $\alpha$, IL-6, IL-12p40, and GM-CSF [28], also in conjunction with IFN- $\gamma$ stimulates macrophages type M1, whose sustained activation triggers a set of inflammatory pathologies as destruction of tissues and encephalitis that takes place in the Central Nervous System (CNS).

The appearance of inflammatory cytokines such as IFN- $\gamma$ and TNF- $\alpha$ favor movement of trypanosomes from the blood to the CNS, process that is associated with the final stages of African trypanosomiasis. During the initial stages of the disease, PLC causes the release of VSG of the membrane of the trypanosomes, this origin Th1 cell response and formation of IFN- $\gamma$; nonetheless, during an advanced stage of this infection, continuous and sustained release of these proteins avoid the activation and signaling of macrophages [29,30]. Infections of mouse with wild type parasites demonstrate different patterns of disease severity, which indicates that the appearance of an acute or chronic infection is controlled by the change of response type 1 (M1 macrophage activation) to response type 2 (M2 regulatory macrophage activation), together with the formation of IL-10 and other anti-inflammatory mediators [31].

\section{Evasion of the adaptive response}

\section{Antigenic variation}

The persistence of T. brucei in the blood is possible due to its capacity of perform the mechanism of antigenic variation, a spectacular process that allows to evade the attack of the host's humoral immune system, that consists in the periodically switching of their major variant surface glycoprotein (VSG), that shield the cell surface from immune effectors [32]. VSG forms a physical coat barrier that protects plasma membrane components of the parasite from exposure to innate and adaptive immune attack. In the course of infection in the mammalian they are produced VSG specific antibodies that eliminate some parasites, others escape from recognition of the antibodies by switching expression to antigenically distinct VSGs, that is to say, "they change their shirts" to avoid being recognized (Figure 2) [33].

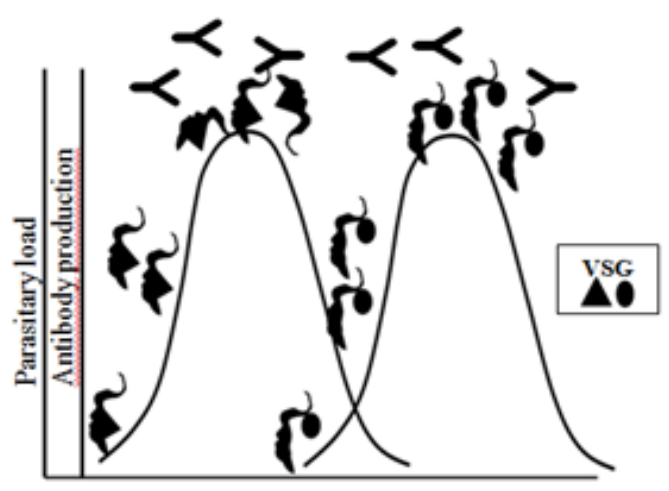

Figure 2. Antigenic variation of T. brucei.

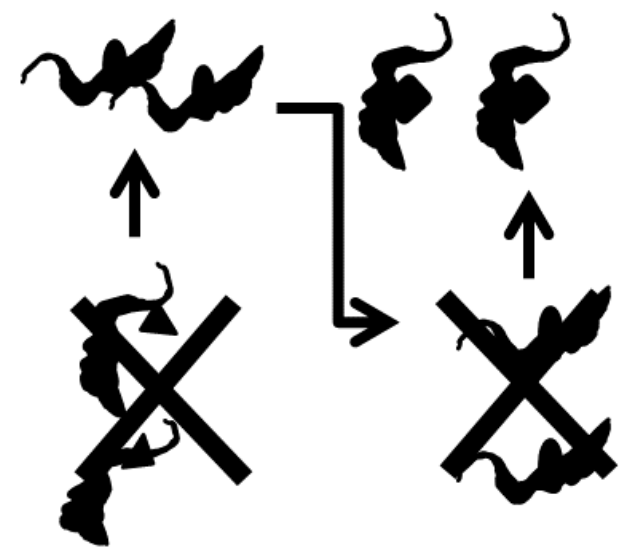

Figure 3. Parasites multiply and suppressed by formation of antibodies against the new antigens present on surface of $T$. brucei.

This layer of VSG constitutes an excellent antigen, because is capable of stimulating an immune response (specifically activating lymphocytes) and provoke the production of antibodies directed against VSG. It results in marking of the pathogen become ingested and eliminated by phagocytes (opsonization) and complement mediated lysis of the most parasites, but some survive because of the modification of their VSG coat, parasites multiply again and are suppressed by the 
formation of antibodies against the new antigens presents on the surface of T. brucei (Figure 3) [34].

These mechanisms of expression and dynamic replacement of VSG are a classic example of adaptive evolution at the hostpathogen interaction. The transcription begins at the same time in all VSG ESs (VSG expression sites), but only one expression site is fully active at a time, that encodes the current coat homogeneous, that is composed of about $10^{7}$ VSGs by parasite. The others are silenced from a genomic repertoire of around 2000, this is because each VSG is encoded by a separate gene $[35,36]$.

\section{Humoral response}

Because AT are extracellular it is logic to think that it occurs a preponderant humoral response, with a marked polyclonal $\mathrm{B}$ cell activation, demonstrated by an increment in the amount of B cells and an evident increment of the quantity of IgG in plasma [37,38]. The antibody produced are polyspecific or auto-reactive [39,40].

During early infection $T$. brucei leads to a decrease of the response of humoral type, this impact over B cells occurs at distinct points: a) it shoots a non-specific, polyclonal B-cell activation, b) take place elimination of the splenic B cell, this can be verified through the decreased of marginal zone and follicular B cell compartments, c) elimination of FoBs mediated by IFN- $\gamma$, this produces a loss of $\mathrm{B}$ cell responsiveness and eliminate protective memory responses, $\mathrm{d}$ ) during the infection of $T$. brucei the B lymphopoiesis is truncated by apoptosis in the bone marrow and spleen already at the level of transitional B cells $[41,42]$.

The antibody-mediated trypanotolerance it is supported by specific antibodies directed against the trypanosome VSG, who are involved in the destruction of trypanosomes in periodic parasitemic waves [43]. Studies realized with animals immunized with VSG or irradiated trypanosomes have been detected protection of the infection with homologous parasites [44]. The antibodies produced specifically against VSG opsonize the parasites, a signaling that allows fagocytosis and elimination by the macrophages [45].

In the process of infection by AT the responses of B cells against VSG may generate in a form T-cell independent [46]. The initial stage of the AT infection is characterized by the formation of specific antibodies against trypanosome that destroy some parasites; however, there is a high percentage of polyspecific antibodies [47]. During the course of late infection the B-cells go through a process of deregulation, where they are removed, that leads to a removal of $\mathrm{IgG}$ responses and greatly decreased IgM response [48].

\section{Antibody clearance}

In addition to antigenic variation, the trypanosomes redistributed surface bound antibodies to the flagellar pocket, in a process known as capping [49]. The VSG coat acts as a physical barrier, serves to protect invariant surface proteins from components of immune effectors [50]. The trypanosomes performed a process of endocytosis that origin the elimination of complex antibody/VSG and allows the evasion of the attack of defense system and the multiplication of parasites in blood.

The VSG cover is internalized using the endocytic process in a short time lapse, and this mechanism occurs in the flagellar pocket [51]. VSG-antibodies are taken inside the parasite; the antibodies are transported to the lysosome and later destroyed [52]. VSG are selected from the rest of proteins in the endosome, apparently through of sorting of GPI-anchored proteins to the recycling pathway, this favors the removal of antibodies of the trypanosome coat. The complex antibodybound VSG prevents the activation of the complement and ultimately the membrane attack complex, thus avoiding the lysis of the parasites [53].

\section{Immunosuppression}

The infections generated by AT produce an amazing immunological suppression, that induce wide deregulation of humoral and cellular functions, mediated by B cell, T-cell and macrophages, respectively, which triggers a series of pathological disorders characteristic of African trypanosomiasis $[54,55]$.

According to some investigations, macrophages and $\mathrm{T}$ cells effect the immunosuppression, during this process can observe blocking of the $\mathrm{T}$ cell multiplication through the elimination of the IL-2 apparition and production of IL-2 receptor [56,57]. $T$. brucei infection inhibits the presentation of antigen of MHC class II, which generates a decrease in the activation of $\mathrm{T}$ cells [58]. In the advanced stage of the disease, macrophages induce the appearance of anti-inflammatory cytokines, such as IL-10 that suppress the presentation of antigen and alter the activation of T cells [59].

\section{Conclusions}

T. brucei is a fabulous and surprising microorganism, which has developed numerous tools to escape the immune defense system of their vertebrate host. The survival of these parasites in mammalian bloodstream depend on an extremely sophisticated strategy denominated antigenic variation, an essential event through which they switch periodically the VSG coat, that is, "they change their shirts" to avoid the destruction mediated by host' humoral response.

The attack of antibodies anti-VSG causes the death of some parasites, instead others survive because they switch the VSG cover by other different that is not recognized by the antibodies produced in that moment; for this reason, AT have been considered masters of disguise. These parasites dramatically modify the activation of macrophages and other antigen presenting cells, what induces to a production of Th1 proinflammatory cytokine as TNF- $\alpha$, IL- 6 and NO production. The VSG of these parasites provoke an imbalance in the profile of cytokines, evidenced by overproduction TNF- $\alpha$ by macrophages, that is to say, the trypanosomes manipulated the effector functions of immune system for its convenience and survival in the mammal, which allows its transmission to the vector insect and thus complete its life cycle. 


\section{References}

1. http://www.cdc.gov/parasites/sleepingsickness/ biology.html.

2. Kennedy P. Clinical features, diagnosis, and treatment of human African trypanosomiasis (sleeping sickness). Lancet Neurol. 2013;12:186-94.

3. Buguet A, Bisser S, Josenando F, et al. Sleep structure: a new diagnostic tool for stage determination in sleeping sickness. Acta Trop. 2005;93:107-17.

4. Jordan A. Tsetse flies as vectors of trypanosomes. Vet Parasitol. 1976;2:143-52.

5. Vincendeau P, Bouteille B. Immunology and immunopathology of African trypanosomiasis. An Acad Bras Cienc. 2006;78:645-65.

6. Diffley P. Trypanosomal surface coat variant antigen causes polyclonal lymphocyte activation. J Immunol. 1983;131:1983-6.

7. Field M, Carrington M. The trypanosome flagellar pocket. Nat Rev Microbiol. 2009;7:775-86.

8. Raper J, Portela M, Lugli E, et al. Trypanosome lytic factors: novel mediators of human innate immunity. Curr Opin Microbiol. 2001;4:402-8.

9. Lecordier L, Uzureau P, Tebabi P, et al. Identification of Trypanosoma brucei components involved in trypanolysis by normal human serum. Mol Microbiol. 2014;94:625-36.

10. Pays E, Vanhollebeke B, Uzureau P, et al. The molecular arms race between African trypanosomes and humans. Nat Rev Microbiol. 2014;12:575-84.

11. Uzureau P, Uzureau S, Lecordier L, et al. Mechanism of Trypanosoma brucei gambiense resistance to human serum. Nature. 2013;501:430-4.

12. Capewell P, Clucas C, DeJesus E, et al. The TgsGP gene is essential for resistance to human serum in Trypanosoma brucei gambiense. PLoS Pathog. 2013;9:e1003686.

13. Vanhamme L, Pays E. The trypanosome lytic factor of human serum and the molecular basis of sleeping sickness. Int J of Parasitol. 2004;34:887-98.

14. Xong $\mathrm{H}$, Vanhamme L, Chamekh $\mathrm{M}$, et al. A VSG expression site-associated gene confers resistance to human serum in Trypanosoma rhodesiense. 1998;95:839-46.

15. Van Meirvenne N, Magnus E, Janssens P. The effect of normal human serum on trypanosomes of distinct antigenic type (ETat 1 to 12) isolated from a strain of Trypanosoma brucei rhodesiense. Ann Soc Belg Med Trop. 1976;56:55-63.

16. Ferrante A, Allison A. Alternative pathway activation of complement by African trypanosomes lacking a glycoprotein coat. Parasite Immunol. 1983;5:491-8.

17. Devine D, Falk R, Balber A. Restriction of the alternative pathway of human complement by intact Trypanosoma brucei subsp. gambiense. Infect Immunol. 1986;52:223-9.

18. Matthews K, McCulloch R, Morrison L. The within-host dynamics of African trypanosome infections. Philos Trans R Soc Lond B Biol Sci. 2015;370: 20140288.
19. Balber A, Bangs $\mathrm{J}$, Jones $\mathrm{S}$, et al. Inactivation or elimination of potentially trypanolytic, complementactivating immune complexes by pathogenic trypanosomes. Infect and Immun. 1979; 24:617-27.

20. Russo D, Williams D, Grab D. Mechanisms for the elimination of potentially lytic complement-fixing variable surface glycoprotein antibody-complexes in Trypanosoma brucei. Parasitol Res. 1994;80:487-92.

21. Van Velthuysen M, Mayen A, Prins F, et al. Phagocytosis by glomerular endothelial cells in infection-related glomerulopathy. Nephrol Dial Transplant. 1994;9:1077-83.

22. Roper J, Guther M, Milne K, et al. Galactose metabolism is essential for African sleeping sickness parasite Trypanosoma brucei. Proc Natl Acad Scie U S A. 2002;99:5884-9.

23. Hovel-Miner G, Mugnier M, Papavasiliou F, et al. A hostpathogen interaction reduced to first principles: antigenic variation in $\mathrm{T}$. brucei. Results Probl Cell Differ. 2015;57:23-46.

24. Grab D, Webster P, Ito S, et al. Subcellular localization of a variable surface glycoprotein phosphatidylinositolspecific phospholipase-C in African trypanosomes. J Cell Biol. 1987; 105:737-46.

25. Paulnock D, Coller S. Analysis of macrophage activation in African trypanosomiasis. $\mathrm{J}$ Leukoc Biol. 2001;69:685-90.

26. Magez S, Stijlemans B, Baral T, et al. VSG-GPI anchors of African trypanosomes: their role in macrophage activation and induction of infection-associated immunopathology. Microbes Infect. 2002:4:999-1006.

27. Leppert B, Mansfield J, Paulnock D. The soluble variant surface glycoprotein of African trypanosomes is recognized by a macrophage scavenger receptor and induces I kappa B alpha degradation inde-pendently of TRAF6- mediated TLR signaling. J of Immunol. 2007;179:548-56.

28. Tachado S, Gerold P, Schwarz R, et al. Signal transduction in macrophages by glycosylphosphatidylinositols of Plasmodium, Trypanosoma, and Leishmania: activation of protein tyrosine kinases and protein kinase $\mathrm{C}$ by inositolglycan and diacylglycerol moieties. Proc Natl Acad Sci, U S A. 1997;94:4022-7.

29. Mansfield J, Paulnock D. Genetic manipulation of African trypanosomes as a tool to dissect the immunobiology of infection. Parasite Immunol. 2008;30:245-53.

30. Namangala B. How the African trypanosomes evade host immune killing. Parasite Immunol. 2011;33:430-7.

31. Tracey L. Immunity to parasitic infection. WileyBlackwell. 2012;520.

32. Morrison L, Marcello L, McCulloch R. Antigenic variation in the African trypanosome: molecular mechanisms and phenotypic complexity. Cell Microbiol. 2009;11:1724-34

33. Dagenais T, Freeman B, Demick K, et al. Processing and presentation of variant surface glycoprotein molecules to $\mathrm{T}$ 
cells in African trypanosomiasis. J Immunol. 2009; 183:3344-55.

34. Zambrano-Villa SD, Rosales-Borjas D, Ortiz-Ortiz L. Mechanisms used by some parasitic protozoa to evade the immune response. Res and Rev in Parasitol 1999;59(3-4): 71-83.

35. Jackson A, Berry A, Aslett M, et al. Antigenic diversity is generated by distinct evolutionary mechanisms in African trypanosome species. Proc Natl Acad Sci U S A. 2012;109:3416-21.

36. Vanhamme L, Poelvoorde P, Pays A, et al. Differential RNA elongation controls the variant surface glycoprotein gene expression sites of Trypanosoma brucei. Mol Microbiol. 2000;36:328-40.

37. Hudson K, Byner C, Freeman J, et al. Immunodepression, high IgM levels and evasion of the immune response in murine trypanosomiasis. Nature. 1976;264:256-8.

38. Luckins A, Mehlitz D. Observations on serum immunoglobulin levels in cattle infected with Trypanosoma brucei, T. vivax and T. congolense," Ann Trop Med Parasitol. 1976;70:479-80.

39. Buza J, Sileghem M, Gwakisa P, et al. CD5+ B lymphocytes are the main source of antibodies reactive with non-parasite antigens in Trypanosoma congolenseinfected cattle. Immunol. 1997;92:226-33.

40. Williams D, Taylor K, Newson J, et al. The role of antivariable surface glycoprotein antibody responses in bovine trypanotolerance. Parasite Immunol. 1996;18:209-18.

41. Bockstal V, Guirnalda P, Caljon G, et al. T. brucei infection reduces $\mathrm{B}$ lymphopoiesis in bone marrow and truncates compensatory splenic lymphopoiesis through transitional B-cell apoptosis. PLoS Pathog. 2011;7:e1002089.

42. Radwanska M, Guirnalda P, De Trez C, et al. Trypanosomiasis-induced B cell apoptosis results in loss of protective anti-parasite antibody responses and abolishment of vaccine-induced memory responses. PLoS Pathog. 2008;4:e1000078.

43. Uzonna J, Kaushik R, Gordon J, et al. Cytokines and antibody responses during Trypanosoma congolense infections in two inbred mouse strains that differ in resistance. Parasite Immunol. 1999;21:57-71.

44. Morrison W, Black S, Paris J, et al. Protective immunity and specificity of antibody responses elicited in cattle by irradiated Trypanosoma brucei. Parasite Immunol. 1982;4:395-407.

45. Pan W, Ogunremi O, Wei G, et al. CR3 (CD11b/CD18) is the major macrophage receptor for IgM antibody-mediated phagocytosis of African trypanosomes: diverse effect on subsequent synthesis of tumor necrosis factor alpha and nitric oxide. Microbes Infect. 2006;8(5):1209-18.

46. Reinitz D, Mansfield J. T-cell-independent and T-celldependent B-cell responses to exposed variant surface glycoprotein epitopes in trypanosome-infected mice. Infect and Immun. 1990;58:2337-42.

47. Kobayakawa $\mathrm{T}$, Louis J, Izui $\mathrm{S}$, et al. Autoimmune response to DNA, red blood cells, and thymocyte antigens in association with polyclonal antibody synthesis during experimental African trypanosomiasis. J Immunol. 1979;122:296-301.

48. Hudson K, Terry R. Immunodepression and the course of infection of a chronic Trypanosoma brucei infection in mice. Parasite Immunol. 1979;1:317-26.

49. Barry J. Capping of variable antigen on Trypanosoma brucei, and its immmunological and biological significance. J Cell Sci. 1979;37:287-302.

50. Horn D. Antigenic variation in African trypanosomes. Mol Biochem Parasitol. 2014;195:123-9.

51. Engstler M, Thilo L, Weise F, et al. Kinetics of endocytosis and recycling of the GPI-anchored variant surface glycoprotein in Trypanosoma brucei. J Cell Sci. 2004; 117:1105-15.

52. Pal A, Hall B, Jeffries $T$, et al. Rab5 and Rab11 mediate transferrin and anti-variant surface glycoprotein antibody recycling in Trypanosoma brucei. Biochem J. 2003;374:443-51.

53. Manna P, Boehm C, Leung $\mathrm{K}$, et al. Life and times: synthesis, trafficking, and evolution of VSG. Trends Parasitol. 2014;30: 251-8.

54. Taylor K. Immune responses of cattle to African trypanosomes: protective or pathogenic? Int J Parasitol. 1998;28:219-40.

55. Sileghem M, Darji A, De Baetselier P. In vitro simulation of immunosuppresion caused by Trypanosoma brucei. Immunol. 1991;73:246-8.

56. Tabel H, Wei G, Shi M. T cells and immunopathogenesis of experimental African trypanosomiasis. Immunol Rev. 2008;225:128-39.

57. Sileghem M, Darji A, Hamers R, et al. Dual role of macrophages in the suppression of interleukin 2 production and interleukin 2 receptor expression in trypanosome-infected mice. Eur J Immunol. 1989;19:829-35.

58. Namangala B, Brys L, Magez S, et al. Trypanosoma brucei brucei infection impairs MHC class II antigen presentation capacity of macrophages. Parasite Immunol. 2000;22: 361-70.

59. Ding L, Linsley P, Huang L, et al. IL-10 inhibits macrophage costimulatory activity by selectively inhibiting the up-regulation of B7 expression. J Immunol. 1993;151:1224-34.

\section{*Correpondence to:}

María Elizaberh Márquez-Contreras

Laboratorio de Enzimología de Parásitos (LEP)

Facultad de Ciencias

Universidad de Los Andes

Mérida-Venezuela

E-mail: emarquez@ula.ve 